\title{
Restructuring Loosely Structured Databases for Generating Local Statistics
}

\author{
John Patrick V.J. Albacea* and Jaime M. Samaniego \\ Institute of Computer Science, College of Arts and Sciences (CAS), University of the Philippines Los Baños (UPLB), \\ Laguna 4031,Philippines; jvalbacea@up.edu.ph, jmsamaniego2@up.edu.ph
}

\begin{abstract}
Objective: Local-level statistics are generated from different data sources for planning and decision-making. A strategy is needed to restructure existing databases in order to construct an integrated database for generating statistics. Methods/ analysis: To achieve this objective, data structures of existing nation-wide surveys and census were studied, and useful data warehousing techniques in combining these data sources for constructing an integrated database were identified. The steps undertaken to build an integrated database were consolidated to formulate the needed strategy. To illustrate the veracity of the strategy, Philippine nation-wide survey and census databases were combined and local-level statistics were generated for exploratory data analysis. Findings: The study was able to identify features of existing databases that were used for the database restructuring and building of an integrated database. Likewise, the study was able to identify the lowest level unit of observation to which data could be disaggregated which will also serve as basis for combining different data sources. From the learning's using some of the Philippine nation-wide surveys and census, the study has developed a strategy to access and combine different nation-wide databases that are physically separated but are related to each other in terms of their data architecture. This led to building an integrated database which could be a source for the generation of statistics that are useful in exploratory data analysis. The results of this analysis are useful for planning and decisionmaking. Furthermore, the study was able to identify ways to warehouse the generated statistics across time and space for future data analysis. Such ways were also incorporated in the developed strategy. Novelty/improvement: The formulated strategy of combining different databases into an integrated database as a source for generating local statistics is scalable to more databases for a richer set of generated statistics.
\end{abstract}

Keywords: Statistical Computing, Data Warehousing, Statistical Integrated Database, Combined Data Sources, Locallevel Statistics

\section{Introduction}

Data are collected, analysed, and then turned into statistics to become useful in planning and decisionmaking processes. Data could be collected through surveys, censuses, registries, administrative records, or even through internet resources.

In addition, data are collected for specific purposes. Once collected, data are transformed into an electronic format and stored in a database. Because of the different ways to collect as well as their different purposes, the structure of their databases also tends to be different.
Censuses are conducted to collect data for each individual or unit of observation under study. In the Philippines, population censuses are conducted by the Philippine Statistics Authority (PSA) every ten years to obtain characteristics of every Filipino resident of the country. The PSA also conducts nationwide surveys for specific purposes. For example, Labour Force Survey (LFS) is being conducted by PSA for employment statistics while Family Income and Expenditure Survey (FIES) is conducted every three years for income and expenditure data to be used for generation of poverty statistics. The unit of observation in the LFS is an individual while it is a

*Author for correspondence 
household in FIES. Registries usually have an individual as the unit of observation like a Cancer Patients Registry but there are other registries which have a group of individuals as unit of observation like the Registry of Cooperatives where an organisation is the unit of observation.

A data set collected from a particular source is stored in a database with a structure, conforming to the unit of observation. Thus, with this scenario, data sets from different sources do have different structures. But these data sets need to be combined to generate more detailed statistics which are needed by the local government in their planning and decision-making processes. For example, FIES data set could be combined with LFS data set and census data to generate poverty statistics at local government level like municipality and city level.

At present, data sets are combined when there is a need to generate municipal and city level statistics and the process of combining data sets is repeated for another statistic to be generated. Hence, the task becomes tedious as the integrated database is not easily available. A remedy to this problem is to have an integrated database at hand that is easy to maintain and update from databases with different structures.

Likewise, there is a need for a data warehouse that could store and maintain a big compiled data set that comes from several major database systems. A data warehouse is different from a data set that was developed and created for a specific purpose and such data set follows the concept of operational data. Nationwide data sets usually follow the concept of operation data. But what is needed in an integrated database is a data warehouse. This is supported in the discussion given in the Data Warehouse Concepts by the Office of the Institutional Research and Academic Planning at Rutgers University. 1 The comparison between operational data and a data warehouse suggests that the latter is more appropriate for an integrated database.

From the same literature, it was stated that there are two main processes in designing a data warehouse. The first process is to determine the required information of the system and its metrics while the second process is the actual development process which is iterative in nature. In this process, a better version of the data warehouse system is produced in every iteration. The iteration continues until the final version of the warehouse is acceptable to the users of the system.

Once the design of the data warehouse was determined, the next step is to populate it with the required information. There are three steps to do this and these steps include extraction, transformation, and loading data. Extraction includes taking all the data out of the system before going to the next step of transformation.

In this step, the idea is to change any information that needs updating and consequently fix any and all anomalies that might have or may occur during the transformation of data. Finally, the transformed information will then be placed back into the data warehouse which can be labelled as loading back the data to the warehouse.

With a data warehouse, mining it to generate statistics for planning and decision making becomes much easier than working on different individual data sets. The process of data mining according to Ref. $\underline{2}$ depends on the type of relationship that exists among the data in the system. The different types are labelled as a class, cluster, association, or in sequential pattern. A relationship that is classified as a class is when the information extracted is based on the previously stored data. An example is using the information in the customer's purchases in a restaurant chain to formulate some daily specials for a bigger profit. The second type of relationship is a cluster. Clusters are groups that are determined by their logical relationships or consumer preference, like when the data are organized in such a way that market segments or consumer affinities can be determined from them. The third type of relationship that can be considered when arranging information is an association. Association, as the name pertains to, collects and arranges data in such a way that the associations among the different data values are readily seen. An example is arranging different products in a store and having products that are normally sold together closer to each other for the convenience of the customer. The last type of relationship is the presence of sequential patterns. An example for this is using information that is mined to predict what the customer will be purchasing next based on the purchases that he or she has already made. Once the relationship of the data is determined, information and statistics processed and then presented for visualisation to obtain the desired outputs.

Using these concepts in data warehousing and data mining, this study aims to present a strategy that could result to an efficient way of combining different database systems for an integrated database that could be warehoused and use the information in the database to generate local level statistics. 


\section{Structures of Some Existing Database Systems in the Country}

\subsection{Population Census Data Set}

A population census data set has an individual resident of the country as the unit of observation. Demographic characteristics of the individual serve as the variables in the data set. These demographic characteristics include the geographical location of the residence of the individual as well as the basic information like sex, age in years as of last birthday, relationship to the household head, disability, and birth registration. Most of the data are qualitative in nature, but all data are stored in numeric format since numeric codes are used instead of the qualitative values.

In the Philippines, the decennial census covers not only the people, but it also covers the housing units in the country that is why it is referred to as Census of Population and Housing or $\mathrm{CPH}$. Thus, the $\mathrm{CPH}$ of the country can be considered to have two data systems based on their units of observations. One system is with an individual person as a unit of observation while the other system has a housing unit as the unit of observation.

Mid-Decade Censuses are also conducted in the Philippines but this time it covers only population census, which is referred to as the Census of Population or CP by the PSA.

In 2015, PSA conducted the most recent $\mathrm{CP}$ while in 2010; PSA conducted the most recent CPH. Some of the data items on each individual as the unit of observation in the census data sets are identified in the following Table 1 with corresponding structure.

\subsection{Nationwide Survey Data Set}

Nationwide surveys are conducted for specific purposes. It takes only representative sample units of observations. The unit of observation may be different from one survey to another. Some surveys have household as the unit of observation while others may have an individual person or an establishment as the unit of observation. PSA is also the government agency mandated to conduct nationwide surveys. PSA uses a master sample for its integrated household surveys. Two of the surveys being conducted by PSA, which were considered in this study, are described below.
Table 1. Structure for some variables in the $2010 \mathrm{CPH}$ data file

\begin{tabular}{|l|l|l|}
\hline Name & Label & Type \\
\hline REGION & Region (coded) & Discrete \\
\hline PROV & Province (coded) & Discrete \\
\hline MUN & City/municipality (coded) & Discrete \\
\hline BGY & Barangay (coded) & Discrete \\
\hline HUSN & Housing unit serial number (coded) & Discrete \\
\hline HSN & Household serial number (coded) & Discrete \\
\hline P1 & Line number & Discrete \\
\hline P2 & $\begin{array}{l}\text { Relationship to household head } \\
\text { (coded) }\end{array}$ & Discrete \\
\hline P3 & Sex (coded) & Discrete \\
\hline P5 & Age (in years) as of last birthday & Discrete \\
\hline P6 & Birth registration (coded) & Discrete \\
\hline
\end{tabular}

1. The Family Income and Expenditure Survey (FIES) is a nationwide survey of households undertaken every three years by the National Statistics Office (NSO). It is the main source of data on family income and expenditure, which include among others, levels of consumption by item of expenditure as well as sources of income in cash and in kind. As NSO (now part of PSA) describes it, the results of FIES provide information on the levels of living and disparities in income of Filipino families, as well as their spending patterns. The 2015 FIES used the master sample designed to provide income and expenditure data that are representative of the country and its 17 regions.

Some of the variables included in FIES data set are information on the characteristics of the household head like his/her age, marital status, sex, highest educational attainment, and other demographic characteristics. Since this is a household survey, the characteristics of the household in terms of its composition were also observed. Likewise, the income and expenditure variables were observed at the household level, that is, total household income and total household expenditure on specific commodities. Some characteristics of the housing unit of the household were also included in the set of data items of FIES. Table 2 presents some of the data items and its structure in FIES data set. There are more than 300 variables being observed in FIES. $\underline{3}$

2. The Labor Force Survey (LFS) is another survey which is being conducted by PSA every quarter of the year. 
Table 2. Structure for some variables in a FIES data file

\begin{tabular}{|l|l|l|}
\hline Name & Label & Type \\
\hline REGN & Region (coded) & Discrete \\
\hline PRV & Province (coded) & Discrete \\
\hline BGY & City/municipality (coded) & Discrete \\
\hline EA & Enumeration area (coded) & Discrete \\
\hline SHSN & $\begin{array}{l}\text { Sample household serial } \\
\text { number (coded) }\end{array}$ \\
\hline SEX & $\begin{array}{l}\text { Sex of the household head } \\
\text { (coded) }\end{array}$ & Discrete \\
\hline AGE & $\begin{array}{l}\text { Age (in years) of the household } \\
\text { head as of last birthday }\end{array}$ & Discrete \\
\hline HGC & $\begin{array}{l}\text { Highest grade computed of the } \\
\text { household head (coded) }\end{array}$ & Discrete \\
\hline TOTEX & $\begin{array}{l}\text { Household annual total } \\
\text { expenditure }\end{array}$ & Continuous \\
\hline TOINC & Household annual total income & Continuous \\
\hline AGELESS5 & $\begin{array}{l}\text { Total number of household } \\
\text { members aged 5 less than 5 } \\
\text { years }\end{array}$ & $\begin{array}{l}\text { Roof material of the housing } \\
\text { unit of the household (coded) }\end{array}$ \\
\hline DOFcrete \\
\hline
\end{tabular}

LFS aims to provide a quantitative framework for the preparation of plans and formulation of policies affecting the labor market. According to PSA, the survey is designed to provide statistics on levels and trends of employment, unemployment and underemployment for the country, as a whole, and for each of the administrative regions, including provinces and key cities.

This survey includes variables for a household member aged 15 years and above, like relationship to household head, age in years as of last birthday, marital status and highest grade completed. Among these household members, those who were employed were asked about their main activity/usual occupation during the reference period, primary occupation, kind of business, class of worker, nature of employment, normal working hours per day during the past week, total hours worked during the past week and whether he/she wanting more hours of work. On the other hand, those who had no job/business were asked about their job search method and number of weeks looking for work. ${ }^{4}$ See Table 3 for the some of the data items and its structure in LFS data set.

\section{Administrative Records}

Table 3. Structure for some variables in an LFS data file

\begin{tabular}{|l|l|l|}
\hline Name & Label & Type \\
\hline REGN & Region (coded) & Discrete \\
\hline PRV & Province (coded) & Discrete \\
\hline MUN & City/municipality (coded) & Discrete \\
\hline BGY & Barangay (coded) & Discrete \\
\hline EA & Enumeration area (coded) & Discrete \\
\hline SHSN & $\begin{array}{l}\text { Sample household serial number } \\
\text { (coded) }\end{array}$ & Discrete \\
\hline C06_SEX & Sex (coded) & Discrete \\
\hline C07_AGE & Age (in years) as of last birthday & Discrete \\
\hline EMPSTAT & Employment status (coded) & Discrete \\
\hline
\end{tabular}

There are data sets which were collected by some institutions like a government agency. These data are usually based on reports provided by local department level to the national offices. The data sets are used in planning purposes as well as for monitoring of the performances of their constituent units. A good example is the data system of the Department of Education (DepEd) referred to as Basic Education Information System or BEIS. In their data system, the unit of observation is a school under the DepEd. The data items in this system are the characteristics of the school like location or address, district, division, total enrolment per grade or year level, total count of teachers, chairs, and books. This is the source of data in generating the basic education statistics of the country.

Another administrative data are coming from the barangay health centers and government hospitals and summarized by the Department of Health usually at the national, regional, and provincial level. The system called Field Health Services Information System or FHSIS provides basic health indicators like total count of hospitals, health workers, and recipients of vaccines as well as incidences of diseases. With its recent development, the electronic version of the FHSIS with barangays as unit of observation is being developed nationwide.

\section{Registry}

A registry can also be considered as a database which contains information about the observational unit. There are registries of individuals and there are also registries of cooperatives or organisations. Registries are also created for a specific purpose. An example is a registry of cancer patients which contain the basic information about a 
patient related to his/her disease. This kind of registry is usually confidential in nature and is used by medical practitioners and researchers for the purpose of finding a cure for the disease or at least finding ways to prevent the disease.

There are also other registries created to monitor the status and conditions of the members listed in the registry. This kind of registry like the Registry of Farmers is being used by concerned agencies in planning and decisionmaking for programs envisioned to the betterment of the farmers. This registry has also an individual as the unit of observation. Registry of cooperatives and/or associations is also available like the registry of coffee growers' cooperatives and registry of associations of weavers in the country. But this kind of registry does not have individual as the unit of observation, but rather has an association or a cooperative as the unit of observation.

\section{Discussion of the Proposed Strategy for the Integrated Database}

The creation and maintenance of the integrated database is a process that extracts the different information from the different databases that may be useful in the study and consequently combine and condense them so that access to the data values would be fast and easy. Things that were considered in the creation of this integrated database include the compilation of crosschecked information and computation of frequently used values. In the compilation of crosschecked information, one has to get the information from the different databases and crosscheck them with existing entries in the other databases and copying them as is into the integrated database.

In order to do this integration, it is required to first understand how the information is arranged in the source databases. As illustration, three source databases were used in the study. All of these databases have the same structure known as star-shaped architecture which basically means that each of them has a center table where most, if not all of the tables are related to. This architecture is illustrated in the following Figure 1.

Because of this common star-shaped architecture, the different databases could be combined into one big data warehouse. The data warehouse has three main parts, namely: data tables, reference tables, and summary

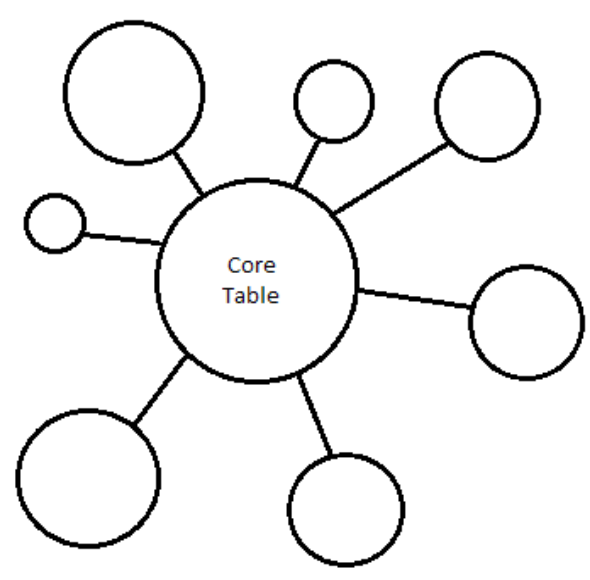

Figure 1. Star-shaped architecture.

tables. The data tables consist of information that was extracted from the database sources. It basically holds a part of the information from the source database as it was laid out in the source database. Secondly, the integrated database has the reference tables which are used to create a uniform and clear coding scheme by using some of the available key features of the source databases. In this way, a standard coding scheme will be provided for the integrated database. Lastly, the summary tables include values that are usually needed by statisticians for data analysis, hence it should be able to handle and maintain the mined data from the source databases. The foreseen data analysis includes the generation of descriptive statistics to be placed in the summary table, the determination of relationship or association among the indicators found in the summary table and visualisation of the statistics generated.

In the construction of the reference table of the integrated database, the core tables of the source databases should be considered. In this study, the three different source databases used to illustrate the strategy include the CPH, FIES, and LFS. To form an integrated database from these three source databases, the center of the architecture should have the core table for $\mathrm{CPH}$, the core table for FIES, and the core table for LFS. The reference tables need to revolve around these three main databases and consequently cater to all of the three core tables. Thus, the main architecture of the integrated database will be something like the one shown in Figure 2 .

In the proposed data architecture of the integrated database, 1-spoke pertains to reference tables that are 


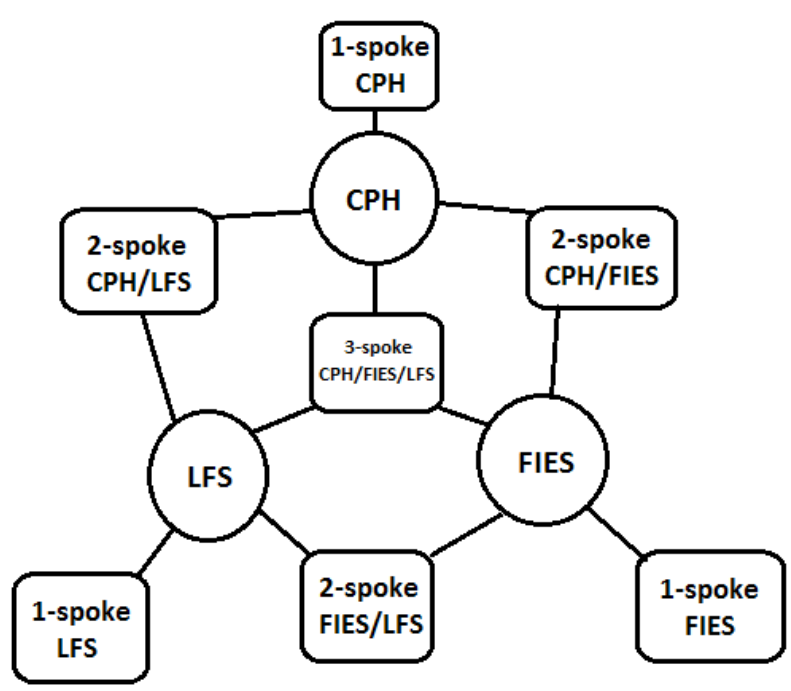

Figure 2. Integrated database architecture.

only used or only cater to one of the three core tables. This implies that in all of the available data in the integrated database, only one of the core databases have this information and therefore, are the only ones that will be using the reference table in the integrated database. Likewise, a 2-spoke pertains to the reference tables that cater to two of the three core tables in the integrated database. This means that if there is a discrepancy between the two databases, these discrepancies will have to be resolved first before it will be able to proceed with the analysis of the information within them. Lastly, 3 -spoke pertains to the reference tables that cater to all of the three core tables in the database. This means that discrepancies among all of the three databases will have to be resolved before analysis can proceed using the variables that use these types of reference tables. By unifying the reference tables in the three source databases, most of this information will be available for the three databases to use and all of the three will not be looking at redundant tables in the database.

In creating uniform reference tables, the common columns and information that are available in the three sources are determined and a uniform set of reference tables for all of them is to be created. With this, all of the data from the three different databases are then placed into one data warehouse with the differences edited so that it will now be able to take the information from the unified reference tables. Examples of the tables that are common in the different databases include the variables labelled as region, province, and sex, relationship to household-head,
FIES indicator, and urban rural classification among others.

As for the tables that needed a transformation before transferring, one of the best examples is found in the Region Table that is available in all of the databases. The $\mathrm{CPH}$ had a different reference table from the other two databases. This implies that it needed to be transformed before it has to be transferred to the integrated database. The two reference tables were both transformed to be uniform, taking the form of the reference table for the $\mathrm{CPH}$. These two reference tables are found in the following Table 4.

With the reference tables transformed, the next to transform is the data table which contains the entries for the three databases. Three tables were created which

Table 4. Region table of the CPH compared to that of FIES and LFS

\begin{tabular}{|c|c|c|}
\hline \multirow{2}{*}{ Region } & \multicolumn{2}{|c|}{$\begin{array}{l}\text { Database name } \\
\text { (value_label) }\end{array}$} \\
\hline & $\begin{array}{c}\mathrm{CPH} \\
\text { (region_id) }\end{array}$ & $\begin{array}{c}\text { FIES and LFS } \\
\text { (w_regn_id) }\end{array}$ \\
\hline $\begin{array}{l}\text { National Capital Region } \\
\text { (NCR) }\end{array}$ & 13 & 13 \\
\hline $\begin{array}{l}\text { Cordillera Administrative } \\
\text { Region (CAR) }\end{array}$ & 14 & 14 \\
\hline Region I - Ilocos Region & 1 & 1 \\
\hline Region II - Cagayan Valley & 2 & 2 \\
\hline Region III - Central Luzon & 3 & 3 \\
\hline $\begin{array}{l}\text { Region IVA } \\
\text { - CALABARZON }\end{array}$ & 4 & 41 \\
\hline Region IVB - MIMAROPA & 17 & 42 \\
\hline Region V - Bicol & 5 & 5 \\
\hline Region VI - Western Visayas & 6 & 6 \\
\hline Region VII - Central Visayas & 7 & 7 \\
\hline $\begin{array}{l}\text { Region VIII - Eastern } \\
\text { Visayas }\end{array}$ & 8 & 8 \\
\hline $\begin{array}{l}\text { Region IX - Zamboanga } \\
\text { Peninsula }\end{array}$ & 9 & 9 \\
\hline $\begin{array}{l}\text { Region X - Northern } \\
\text { Mindanao }\end{array}$ & 10 & 10 \\
\hline Region XI - Davao & 11 & 11 \\
\hline $\begin{array}{l}\text { Region XII } \\
\text { - SOCCSKSARGEN }\end{array}$ & 12 & 12 \\
\hline $\begin{array}{l}\text { Autonomous Region in } \\
\text { Muslim Mindanao (ARMM) }\end{array}$ & 15 & 15 \\
\hline Region XIII - Caraga & 16 & 16 \\
\hline
\end{tabular}


indicated the source of each information. These three tables were extracted from the three databases and were transformed to be placed into the database that is going to be used for the analysis. The transformation includes having all of the entries edited so that the tables will have the correct information within their rows like changing the values of the regions per row in the FIES and the LFS tables. The inclusion of the raw information of the three databases in the data warehouse represents the first part of the two-step process. This first part of the process created a local copy of all the information that are available in the three databases which then gives the ability for the data warehouse to do the computations independent of the database sources.

As for the last part of the integrated database, the focus is the set of computed values from the three databases. This mainly includes simple common derived values such as total number of entries that have a specific value recorded in the tables. Some examples of these computed values are the total number of males and females in the FIES database, the total number of people who are married in one region from the $\mathrm{CPH}$ database, and other simple tally of values. With this kind of derived information available in the integrated database, further computations could be done that might be needed for the analysis part. This part of the integrated database creation represents the second part of the two-step process.

The result of the two-step process is the completed integrated database system which is an input to data warehousing. It contains not only a unified version of the three database sources, but also some derived values and attributes of the three databases. The database in the warehouse is now available for data mining.

Mining the system means traversing the integrated database to create and maintain summary tables in the database. The summary table contains statistics at the smallest unit of observation or lowest level of disaggregation that is common for all source databases. The recommended statistics is in the form of total count or sum of the values. From the sum and/or total count other statistics like average and proportion could easily be computed. For a measure of dispersion or variability, the

Table 5. Variable name and description in the sample summary table

\begin{tabular}{|c|c|c|}
\hline Variable name & Variable description & Source database \\
\hline Regn & Region & CPH/FIES/LFS \\
\hline Prov & Province & CPH/FIES/LFS \\
\hline Mun & Municipality & CPH/FIES/LFS \\
\hline Bgy & Barangay & CPH/FIES/LFS \\
\hline countNG & Total count of persons who did not have formal education & $\mathrm{CPH}$ \\
\hline countEU & Total count of persons who started elementary education but did not graduate & $\mathrm{CPH}$ \\
\hline countEG & Total count of persons who completed elementary education & $\mathrm{CPH}$ \\
\hline countHU & Total count of persons who started secondary education but did not graduate & $\mathrm{CPH}$ \\
\hline countHG & Total count of persons who completed secondary education & $\mathrm{CPH}$ \\
\hline countCU & Total count of persons who started tertiary education but did not graduate & $\mathrm{CPH}$ \\
\hline countCG & Total count of persons who completed tertiary education & $\mathrm{CPH}$ \\
\hline countYes & Total count of persons who are overseas workers & $\mathrm{CPH}$ \\
\hline countNo & Total count of persons who are not overseas workers & $\mathrm{CPH}$ \\
\hline texpendSum & Total household expenditure & FIES \\
\hline texpendSum2 & Sum of squares of household expenditure & FIES \\
\hline tincomeSum & Total household income & FIES \\
\hline tincomeSum2 & Sum of squares of household income & FIES \\
\hline countNES1 & Total count of persons who are employed & LFS \\
\hline countNES2 & Total count of persons who are unemployed & LFS \\
\hline countNES3 & Total count of persons who are underemployed & LFS \\
\hline
\end{tabular}


sum of the square of the values is also recommended to be part of the summary tables.

For the source databases in the illustration, a barangay is the recommended smallest unit of observation or lowest level of disaggregation to use. Total counts are to be computed for discrete variables while the sum of the values and sum of the square of the values are the statistics to be computed at the barangay level. These statistics are then placed in the summary tables which could be used for data analysis. Table 5 contains variables in the summary table, their description and from which database they were derived from.

Using the information in the summary table, descriptive statistics can then be computed. Likewise, the association of the variables could also be explored graphically or even measuring the degree of the association. This information can help planners and/or decision makers in their course of actions to take.

Scatter plots like the plots shown in Figures 3 and 4 illustrate the degree of association between two variables of interest could be generated from data found in the summary tables. For example, based on these scatter plots, the associations shown have direct relationships. Because of this, some claims can be further looked into. For this particular example, one certain claim that may warrant a closer look is the association of having college graduates choosing to go abroad for work and college undergraduates choosing to stay either because they lack

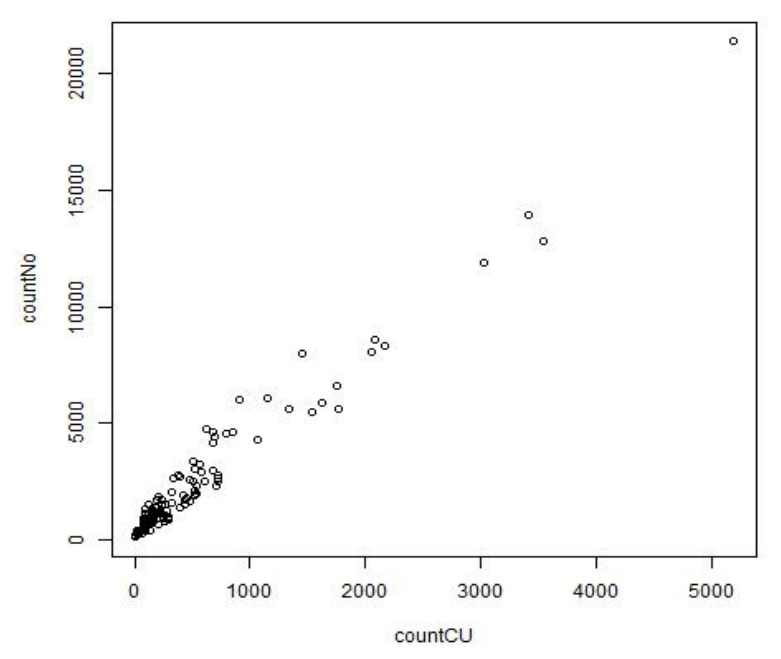

Figure 3. Scatter plot of the total number of college undergraduate and total count of overseas work.

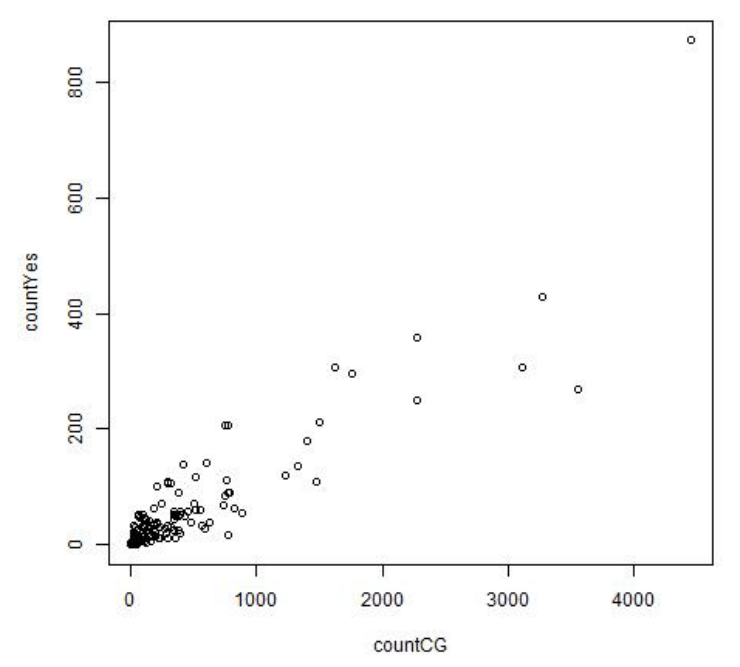

Figure 4. Scatter plot of the total number of college graduate and total count of overseas work.

the skills to be qualified for work abroad or for some other reasons.

Similar to this kind of analysis is what could be done when one has the summary tables obtained from an integrated database. A closer look at the numbers could help planners or decision makers or even government officials in their decision-making process. Having these statistics to base the plan or the decision is an advantage and a well-designed integrated database could support this process are Table 5 .

\section{Conclusion}

With all of the systems designed and implemented, it is safe to say that there is a possible way to integrate and use the information from the different data sources. Using technology that is both free and accessible, the creation and maintenance of the integrated database is plausible and much needed. The benefits it brings are many and the reasons for it to exist support this. The integrated database serves as a tool in organising data to generate statistics. Furthermore, an integrated archive will be useful in the future when it comes to studying what has happened in the past to determine solutions for problems or even predicting trends in the future.

The database itself is a sustainable and maintainable archive of information. Improvements can still be done on different parts of the system. Although, on its own, it is 
a working prototype that can do some of the more tedious tasks to help in the work of different professionals. The main improvement can be placed in either the choice of database management system or the implementation of the management of information.

As for the setting up of the combined data sets for analysis, the summary tables have been created and all of them are catering to the general needs of professionals to explore and use in different types of analysis with regard to the three source databases. The summary tables have paved a way for the professionals to not only create and explore more information, but to also give them a more detailed way of data analysis by setting up the variables using different statistical techniques.

In addition, with the compilation and creation of the visualisations of the data, it is very easy to see that there are a lot of potential in looking at the trends they may show. With the creation of the visualisations, some of the more difficult to see trends can be more visible to the users. With these trends being visible, users will now be able to formulate their own observations and analysis of the data.

\section{References}

1. Data warehouse concepts. [cited 2014]. https://www. tutorialspoint.com/dwh/dwh_data_warehousing.htm.

2. Data mining: what is data mining? [cited 2015 Aug 06]. https:// ucarecdn.com/1bc035bc-0f0e-454e-a9b0-fe72beacd960/

3. Family income and expenditure survey (FIES). [cited 2014]. https://psa.gov.ph/tags/family-income-and-expendituresurvey.

4. Technical notes on labor force survey (LFS). [cited 2012 May 04]. http://www.psa.gov.ph/content/technical-notes-laborforce-survey-lfs 